\title{
Los factores clave del éxito de las promociones realizadas con dispositivos móviles de última generación: un análisis teórico.
}

\author{
David Roman CoY ${ }^{1}$ \\ EADA Escuela de Alta Dirección y Administración \\ Irene GARCíA MEDINA² \\ Universitat de Vic
}

Recibido: $19 / 09 / 2012$

Aceptado: 15/04/2013

\begin{abstract}
Resumen
La utilización de los dispositivos móviles ha crecido exponencialmente en los últimos 15 años llegando a la culminación con la introducción de los teléfonos inteligentes y las tabletas. Las empresas y organizaciones tienen un nuevo canal con múltiples posibilidades comerciales y lo están aprovechando. Analizar esta realidad a través de la literatura académica ha sido el objetivo de esta investigación. Nos ha permitido averiguar por qué las empresas utilizan cada vez más los dispositivos móviles para realizar promociones comerciales y nos ha mostrado los factores clave para realizar promociones exitosas utilizando los terminales móviles de última generación como soporte promocional.
\end{abstract}

Palabras clave: Promociones móviles; Promociones comerciales; Dispositivos móviles; Publicidad móvil; Marketing móvil.

The key success factors in the promotions carried out with last generation mobile devices: a theoretical analysis

\begin{abstract}
The use of mobile devices has grown exponentially in the last 15 years. This development has reached the culmination with the introduction in 2007 of Smartphones and Tablets. Companies and organizations have a new channel with multiple commercial possibilities and are doing the best of it. The objective of this research was to analyze and understand this reality through academic literature. The results of the investigation will clarify the reasons why commercial promotions are successful and it will show us what the most important factors are that make mobile devices extend and improve the way in which companies perform promotional activities.

Key words: Mobile promotions; Sales Promotions; Mobile Devices; Mobile Advertising; Mobile Marketing

${ }^{1}$ Profesor de Marketing en EADA. Miembro del Grupo de Investigación de Interacciones Digitales (GRID) de la Universitat de Vic. Licenciado en Ciencias de la Comunicación (Universitat Autònoma de Barcelona). Máster en Business Administration por EADA. Master en Comunicación Digital Interactiva por la Universitat de Vic. Doctorando en la Universitat de Vic.droman@eada.edu

${ }^{2}$ Profesora titular en la Facultad de Empresa y Comunicación de la Universidad de Vic (Barcelona, España). Miembro del Grupo de Investigación de Interacciones Digitales (GRID) de la Universitat de Vic. Licenciada en Ciencias de la Comunicación (Universidad Complutense de Madrid, España) y Doctora en Marketing (Universidad de Sophia-Antipolis, Francia) y en Relaciones Internacionales (Universidad de Viena, Austria).iregarcia@gmail.com
\end{abstract}




\section{Introducción}

Desde 2006 hay más teléfonos móviles que personas en España ${ }^{3}$. La gran penetración de estos aparatos entre la población española, 58 millones de dispositivos ${ }^{4}$ frente a poco más de 47 millones de habitantes 5 , ha hecho de España uno de los países de la Europa Occidental con mayor penetración de la telefonía móvil. La introducción de dispositivos móviles de última generación ha ampliado de forma notoria las utilidades de estos aparatos convirtiéndolos en un excelente canal de comunicación comercial entre las empresas y los consumidores.

A pesar de que RIM, Palm, Nokia y otros fabricantes ya tenían modelos que, con limitaciones, se podrían llamar de esta forma, no es hasta 2007 en que los teléfonos inteligentes (Smartphones) comienzan a consolidarse entre los usuarios de teléfonos móviles, con el lanzamiento del IPhone de Apple. Estos dispositivos con una pantalla mayor y con sistema operativo móvil, se han convertido en la «auténtica navaja suiza del siglo XXI» (Livingstone, 2004).

Su aceptación por parte de los consumidores es muy grande y, a finales de 2011, ya representaban casi la mitad del parque de teléfonos móviles españoles, el $49 \%{ }^{6}$. A estos dispositivos, tenemos que añadir las tabletas, a medio camino entre un ordenador y un Smartphone que, a pesar de existir desde los años 70, no explotaron comercialmente hasta el año 2010 con el lanzamiento del IPad de Apple. Teléfonos inteligentes y tabletas, suponen un canal de comunicación que incluye múltiples formatos audiovisuales y la portabilidad del dispositivo móvil. Los responsables de marketing y comunicación han visto en este canal una gran oportunidad para comunicar con sus públicos objetivo.

Bajo esta base se sustenta esta investigación, que ha consistido en averiguar los factores clave que aumentan la efectividad en las promociones comerciales y si los dispositivos móviles poseían las características adecuadas para mejorar los resultados de las promociones tradicionales a través de soportes online y offline.

\section{Limitaciones y metodología aplicada}

La gran proliferación de dispositivos móviles y su utilización embrionaria por parte de las organizaciones hace que sea complicado poder seguir reglas establecidas para analizar este campo. La falta de literatura nacional, que tenga como foco las promociones a través de dispositivos móviles, especialmente de última generación, ha llevado a que la revisión de la literatura se haya basado especialmente en autores de fuera de España, concretamente en investigadores norteamericanos, británicos, escandinavos y asiáticos. En estos países la utilización del móvil como soporte de marketing está mucho más arraigada que en nuestro país, y también está más analizada.

\footnotetext{
${ }^{3}$ CMT: Comisión del mercado de las Telecomunicaciones, noviembre de 2006.

${ }^{4}$ CMT: Comisión del mercado de las Telecomunicaciones, diciembre de 2011.

${ }^{5}$ INE, diciembre 2011

${ }^{6}$ AC Nielsen, diciembre 2011.
} 
La variedad de promociones comerciales realizadas dentro del departamento de marketing de la empresa, desde las más comerciales hasta las más relacionadas con el departamento de comunicación, provoca que no sea sencillo separar lo que es específicamente una promoción comercial de una campaña. Tampoco es fácil separar el canal por el mismo motivo, ya que una misma empresa puede utilizar diferentes soportes para enviar el mismo mensaje a diferentes públicos. Ejemplos como éste amplían el foco de la investigación y dificultan la misma.

En esta investigación, no se han podido analizar otras áreas académicas como puede ser el análisis de los comportamientos de compra de los consumidores, ámbitos de psicología del consumo o sistemas de fijación de precios, entre otras. No se ha pretendido hacer extensiva la investigación a estas otras áreas al considerar que supondría un campo de estudio demasiado vasto, aunque son áreas que pueden ser analizadas en posteriores investigaciones sobre el tema.

Tampoco han sido objeto de estudio en este artículo las promociones que sean de carácter no comercial, como promociones públicas, de servicios, de prevención sanitaria... así como tampoco promociones de empresas de telefonía móvil, si éstas no usan los dispositivos móviles como canal de comunicación con sus públicos objetivo.

El planteamiento de investigación se ha basado en revisión de literatura académica sobre el objeto de estudio, principalmente en países en los que el marketing móvil y la realización de promociones a través de estos dispositivos están más avanzados como acabamos de señalar. Para ello se han utilizado técnicas de investigación basadas en una revisión de fuentes bibliográficas y hemerográficas (informes publicados, libros, estudios realizados por fuentes rigurosas y contrastadas). El análisis de literatura ha permitido contrastar, actualizar y complementar datos relevantes (Ruiz - 2007) recopilando información previa existente sobre el objeto de estudio con la finalidad de revisar los marcos teóricos, metodologías y conclusiones a las que han llegado otros investigadores relevantes, permitiendo valorar en qué punto se encuentra el estado de la cuestión en la actualidad y proporcionando análisis objetivos de la literatura experta separando los datos empíricos de la interpretación de sus resultados.

\section{Factores clave del éxito de las promociones realizadas con dispositivos móviles de última generación}

Holmen (2008) y Novikov (2008) desarrollan su investigación afirmando que uno de los factores de éxito del marketing móvil es la eficiencia del soporte frente a otros soportes tradicionales. Por otro lado, otros factores que explican su mayor efectividad son sus posibilidades de personalización y de individualización de contenidos, el alto grado de respuesta y su coste inferior a otros soportes, según Kassavalis, Spyropoulou, Drosos, Mitrokostas, Gikas \& Hatzistamatiou (2003) y Scharl, Dickinger \& Murphy (2005). También cabe tener en cuenta otros factores como el comportamiento del consumidor, la entrega de la información en el momento de la decisión y en el lugar adecuado, la posibilidad de interacción y la posibilidad de feedback inmediato, como se 
destaca en los análisis de Ktoridou, Eparninonda \& Vrontis, (2007).

Uno de los elementos más estudiados académicamente y en claro ascenso son los cupones móviles. Empresas como Groupon o DailyDeals, en formato de grupos de compra, han tenido un gran crecimiento en diferentes países occidentales, incluso llegando la primera a cotizar en bolsa. El cupón es uno de los elementos más conocidos y aceptados tanto por consumidores como por comerciantes. Hay diversas definiciones de cupón. Un cupón proporciona a un comprador una reducción en el precio de un producto o servicio (O'Guinn, Allen \& Semenik, 1998), es una especie de certificado que permite a un consumidor obtener un precio reducido en el momento de la compra (Schultz, Robinson \& Petrison, 1998) o es un certificado que autoriza a su portador a un ahorro en una compra de un producto específico (Kotler \& Armstrong, 2010). Los cupones pueden también usarse para conseguir reintegros, ofertas combinadas, muestras gratuitas u otro tipo de promociones indirectas, como concursos o sorteos (Schultz, Robinson \& Petrison, 1998).

Los cupones online ofrecieron en su momento una mejora importante en cuanto a la distribución y redención. Un consumidor puede acceder a un cupón a través de sitios web o búsquedas, descargárselo e imprimirlo para su redención. Estos cupones también pueden ser enviados directamente a consumidores registrados, normalmente vía correo electrónico o SMS. La buena aceptación general ha provocado que intermediarios de cupones estén comercializando descuentos, como la mencionada Groupon. Los cupones online tienen bastantes ventajas frente a los tradicionales tanto para los comerciantes como para los consumidores. Una ventaja obvia para las empresas es el ahorro en coste y tiempo (Carmody, 2001). Desaparecen los costes de impresión, de distribución y en parte los de creación de base de datos, al ser introducidos en ocasiones por el propio usuario. Desde la perspectiva de los consumidores, los cupones online son también beneficiosos al reducir el tiempo y el esfuerzo necesario para buscar, clasificar y organizarlos (Fortin, 2000).

Para conseguir un alto grado de redención es importante que el cupón sea distribuido correctamente (Neslin \& Clarke, 1987). A través de los cupones online, es posible una segmentación de los consumidores, ofreciendo promociones más adaptadas, por lo que sus ratios de redención acostumbran a ser mayores que los de los tradicionales (Fortin, 2000). Por otro lado, los cupones online presentan una serie de problemas: la falta de control del número de cupones descargados o las reimpresiones de éstos, su posible manipulación por parte de algún usuario y la ineficacia de algunos proveedores de cupones. Además, algunas redenciones demasiado elevadas pueden molestar a los proveedores de cupones y a su rentabilidad (Fortin, 2000). No obstante, cualquier desviación puede ser fácilmente corregida con las promociones a través de dispositivos móviles, que permiten un mayor control de la acción promocional, de forma inmediata y económica. 
La ratio de redención de cupones se calcula dividiendo el número de cupones distribuidos por el número de cupones canjeados (NCH Marketing Services, 2010). El ratio de redención de los cupones offline se calcula dividiendo el número de cupones distribuidos en folletos entre el número de cupones canjeados, mientras que en el caso de online son los cupones descargados entre los canjeados (Jung \& Lee, 2010).

Considerando la naturaleza de Internet y la vasta información a la que se puede acceder, se puede dar por hecho que los usuarios de cupones online pueden encontrar información de otros precios de referencia (Jung \& Lee, 2010), lo que hace el medio online mucho más transparente y competitivo. Siempre que los responsables de marketing de las empresas puedan tener acceso a sus clientes a través de Internet, se recomienda usar cupones online al tener una mayor ratio de redención y una mayor repercusión en las ventas.

\subsection{Factores que ayudan a mejorar las promociones tradicionales utilizando dis- positivos móviles}

Con toda la revisión de la literatura establecida, se ha generado una clasificación que aglutina los resultados de las investigaciones empíricas de otros autores, referida a los factores en que los dispositivos móviles puedan mejorar las promociones tradicionales, establecida en el siguiente decálogo, basado en los trabajos de Yang, Liu \& Zhou (2012) The Chartered Institute of Marketing (2011), Leppäniemi \& Karjalouoto (2005), Kondo, Jian \& Shahriar (2008), Barwise \& Strong (2002), Kondo \& Nakahara (2007), Tsang, Ho \& Lian (2004), Wiedemann, Walsh \& Mitchell (2008), Godin (2001), Clickatell (2003) y Green (2012) como base principal.

1. El puente entre la realidad offline y la oferta online que los dispositivos móviles tienden tanto a empresas como a consumidores.

2. La localización, que permite hacer promociones no únicamente por criterios de segmentación de perfil de usuarios, sino por su ubicación en un instante preciso.

3. La minería de datos y el registro del comportamiento de compra permiten a los anunciantes la personalización total mediante mensajes promocionales individualizados.

4. Las aplicaciones para dispositivos móviles permiten la comparación de productos en el mismo punto de venta, lo cual puede determinar su comportamiento de compra y la reacción inmediata de la empresa.

5. Los nuevos medios permiten al consumidor comentar, pedir opinión, consejo, etc. y compartir la información con otros usuarios, en un proceso de socialización del proceso de compra.

6. El registro electrónico de la actividad simplifica el proceso de control y recogida de datos en la redención de las promociones, favoreciendo posteriores campañas más individualizadas.

7. Una nueva posibilidad es la interactividad, ya que la comunicación no es unidireccional, sino que los consumidores pueden dirigirse a su vez a la empresa, comentar y negociar. 
8. La rapidez es una de las características de estos canales donde todo es inmediato. Esto permite respuestas instantáneas y promociones más cortas.

9. Los nuevos canales online tienen unos costes mucho más económicos que los de las campañas tradicionales, suprimiendo costes logísticos, de impresión, etc.

10. Por último, destaca la integración tecnológica, propia del ecosistema móvil, que ofrece diferentes herramientas integradas en el terminal y aumenta la experiencia entre consumidores y empresas.

Analizaremos con más detalle esta clasificación:

El puente entre el offline y el online. Los dispositivos móviles de última generación se han convertido en un aglutinador de la compra tradicional y el e-commerce. La integración de los dos tipos de compra permite compartir lo mejor de las experiencias online con las offline. Capturar información en el punto de venta para consultarla online a través del Smartphone o saber cuál es el comercio que proporciona un mejor descuento en la zona en la que está el cliente a través del dispositivo móvil, abre las puertas a una nueva forma de comerciar, en la que la distancia entre fabricantes y comerciantes se reduce. El consumidor nunca ha estado offline u online, sino que compra en función de su comodidad y momento. Por este motivo, las empresas no pueden separar su oferta, sino integrarla. Seguramente el nuevo término de futuro es el consumidor all-line. Las tiendas físicas tendrán que ser más digitales y las digitales más físicas, sin duda. La ubicuidad de los dispositivos móviles permite a los consumidores expandir contenidos en cualquier sitio y desde cualquier lugar, dando inmediatez a las campañas de marketing (Yang, Liu \& Zhou, 2012).

Localización. La geolocalización permite mejorar las posibilidades que la distribución de folletos ofrecía. Ahora una empresa puede ubicar a un consumidor en una situación determinada y ofrecerle un beneficio extra para que se acerque a su punto de venta a comprar el producto. Una gran manera de segmentar que, además, permite estrategias diferentes para cada momento del día, ya que un plan promocional puede estar vigente únicamente para un día, horas o minutos determinados. La localización personal es el punto clave para las empresas que quieran operar en el marketing móvil. La combinación entre la localización de la persona y la presencia constante en el dispositivo móvil es una mezcla que nos acerca al éxito de la campaña, como han demostrado experiencias como la de la cadena de comida rápida Burger King (The Chartered Institute of Marketing, 2011).

Personalización. El dispositivo móvil se convertirá en un identificador personal. La ubicuidad, movilidad y localización permanente que los dispositivos móviles proporcionan se transforma en el canal óptimo para enviar ofertas personalizadas a consumidores seleccionados, aumentando su efectividad. Una buena estrategia de CRM que cruce diferentes datos capturados de los consumidores debe estar al servicio de la relación con el consumidor, punto básico para conseguir los objetivos comerciales, sin olvidar nunca la premisa de respetar las voluntades del cliente en todo momento, para 
no perder su confianza y derribando, de esta forma, uno de los principales frenos al uso del marketing móvil, la invasión de la intimidad. Las diferentes formas de promociones a través del móvil y en concreto los cupones móviles, incrementaron el tráfico a la tienda, añadieron valor a la compra y crearon posibilidades de personalización de una manera eficiente en cuanto a costes (Kondo, Jian \& Shahriar (2008).

Heionoen y Strandwik (2002) realizaron un estudio empírico que reveló que el servicio de mensajes cortos (SMS) era el medio más irritante para los usuarios de teléfonos, mientras que los medios tradicionales fueron los menos. Un estudio que mide las actitudes de los consumidores hacia la publicidad móvil llegó a la conclusión de que recibir mensajes comerciales fue percibido como perturbador y negativo por los consumidores (Tsang, Ho \& Lian, 2004) y que el teléfono móvil es una parte de nosotros y no reaccionamos bien a las intrusiones (The Chartered Institute of Marketing, 2011). Sin embargo, cuando se recibieron mensajes de texto comerciales con previo permiso de los usuarios, estos crearon actitudes positivas hacia el anunciante basándose en los valores de entretenimiento, información y credibilidad (Tsang, Ho \& Lian, 2004). Los consumidores han visto que la publicidad móvil basada en el permiso es una buena vía de comunicación en pleno desarrollo. El número de envíos es la clave para la aceptación del servicio, como también la relevancia del anuncio publicitario, la frecuencia de la publicidad, la estandarización del texto, la recompensa y el permiso explícito (Barwise \& Strong, 2002). La segmentación por aceptación de la Internet móvil debería ser una consideración básica para los responsables de marketing; cuanto más fácil perciben los consumidores el uso de Internet móvil, más favorable será su respuesta al correo móvil (Kondo \& Nakahara, 2007).

Comparación. A través del terminal móvil se pueden comparar precios entre un punto de venta y otro. El Smartshopper (consumidor inteligente) podrá estar en un punto de venta, escanear el código de producto o simplemente hacerle una fotografía y poder comparar su precio con otros puntos de venta u otros productos sustitutivos. Este será un momento álgido para conseguir retener un cliente o captar uno nuevo, ya que este instante del proceso de compra es el momento de la decisión.

Ahorrar dinero y tener mayor conocimiento del lugar de compra son aspectos muy valorados por los usuarios (Yang, Liu \& Zhou, 2012) y la comparación de precios abre unos amplios horizontes a los sistemas tradicionales de compra, los que entrarán nuevos parámetros de comportamiento del consumidor.

Socialización. La web 2.0 también está presente en el móvil. Los consumidores somos sociales y compartimos y comparamos información. Los consejos de Foursquare mediante crowdsourcing pueden ser decisivos para una elección comercial y, cómo no, se pueden promocionar. El papel del community manager se puede expandir hasta estos territorios, trabajando para conseguir recomendaciones «promocionadas». Los mensajes útiles, con entretenimiento, relevantes y que implican los valores propios se «viralizan» más fácilmente (Yang, Liu \& Zhou, 2012). Los mensajes promocionales a través 
de móviles se caracterizan por su ubicuidad, inmediatez, credibilidad y legitimidad y pueden ser muy eficientes al conseguir diseminaciones exponenciales a un coste muy reducido para los publicistas (Yang, Liu \& Zhou, 2012). Si los mensajes comerciales provienen de familiares o amigos cercanos, los que los reciben los aceptan mejor y son más creíbles que los mensajes comerciales enviados directamente por las empresas (Wiedemann, Walsh \& Mitchell, 2008).

El registro electrónico de la actividad, se ve facilitado por el rastro electrónico que deja cualquier interacción con un teléfono móvil. Este rastro permite un mayor control de las promociones por parte de las empresas, simplificando los procesos y la recogida de los datos en el momento de la redención. Esta funcionalidad permite aprender de las campañas realizadas, consiguiendo posteriores acciones más individualizadas en función de aquellos segmentos, franjas horarias, tamaño de oferta u otras variables que el promotor considere importantes. Así también se consigue, aparte de aumentar la redención, según Neslin \& Clarke (1987), una mayor satisfacción de los usuarios que reciben promociones de mayor valor añadido. Todas estas virtudes se comparten con las promociones online, aunque la utilización individual del dispositivo móvil permite una comunicación más personalizada y un mayor control de la promoción que no la de los mensajes enviados por internet a ordenadores, compartidos por varios miembros de una misma familia, además de otros problemas como la imposibilidad de limitar el número de los cupones descargados o las reimpresiones de éstos y las posibles manipulaciones (Fortin, 2000).

Interactividad. Ahora los consumidores también tienen algo que decir y la comunicación de las empresas no es únicamente a través de sistemas push. Iniciativas que permiten que los consumidores busquen promociones de productos determinados o poder responder a las promociones que se les envían conlleva un nuevo campo de actuación para las empresas, que deben ser capaces también de llevar la conversación. Asegurar que los sitios web de los anunciantes tengan una versión amigable para móviles e incentivar la interactividad es uno de los caminos por los que transcurre el marketing móvil (The Chartered Institute of Marketing, 2011). Sistemas de inteligencia artificial serán seguramente la próxima incorporación a las relaciones con los clientes.

Rapidez. Si uno de los factores que hace que una promoción pueda tener más éxito es la rapidez del premio recibido, las empresas pueden dar premios inmediatos, sin tener que esperar a envíos por correo, personalizados, con un coste fijo inferior al de promociones no virtuales. Un formato similar a como funciona el mercado de la música actual, eliminando el soporte físico por transacciones online (Yang, Liu \& Zhou, 2012).

Economía. Tanto para los usuarios como para los responsables de marketing, las campañas a través de dispositivos móviles de anteriores generaciones son más económicas que las promociones tradicionales y con la llegada de los Smartphones y tabletas, igual de económicas que las promociones online. Esto abre un canal de alto interés para las empresas, aunque con los riesgos del posible spam que debe controlarse para no 
«quemar» el canal, siguiendo las reglas del marketing de permiso (Godin, 2001). También para los usuarios, el poco riesgo del bajo o nulo precio que tienen que pagar por reenviar mensajes y la gratuidad para el que los recibe aumenta su factor viral (Yang, Liu \& Zhou, 2012).

Uso de tecnologías integradas. El modelo de Davis (1989) sobre la Aceptación de la Tecnología (Technology Acceptance Model - TAM) se fundamenta en dos variables, la utilidad percibida de la tecnología y su facilidad de uso, puntos que los dispositivos móviles integran y que explican su rápida aceptación social, demostrada por su rápido crecimiento. Todas las utilidades de los dispositivos móviles pueden estar al servicio de la estrategia de comunicación y comercial de las empresas. Los códigos de respuesta rápida pueden ser un soporte para la publicidad y también para las actividades promocionales de las organizaciones.

Como hemos visto, promoción no es únicamente un descuento en precio o producto, sino cualquier valor añadido que se ofrezca a un consumidor durante un espacio de tiempo limitado. En esta línea, participar en juegos a través del móvil o experiencias de realidad aumentada provocan en las empresas una nueva manera de desarrollar políticas promocionales, en las que se puede juntar un factor lúdico con una recompensa, en un nuevo formato de yincana o concurso (The Chartered Institute of Marketing, 2011). Otra de las tecnologías que pueden cambiar la relación con los consumidores es la NFC (Near Field Communication), especialmente por las posibilidades que abren en cuanto al pago a través de dispositivos móviles. La clave del éxito radicará no en incorporar tecnología, sino en ver que necesidades tienen los usuarios y cómo poner la tecnología a su servicio (The Chartered Institute of Marketing, 2011). La gran cantidad de datos que se acumularán en el momento en que hagamos nuestras compras a través de nuestros dispositivos móviles permitirá que los planes de fidelización puedan estar integrados en estas plataformas de pago, sin necesidad de acarrear tarjetas de fidelización y permitiendo a las empresas buscar políticas de promociones instantáneas o basadas en siguientes compras, alcanzando así no únicamente a los usuarios que siguen un plan de fidelización con la empresa, sino a cualquier cliente.

El objetivo de buscar la máxima redención posible consigue, en las promociones a través del móvil, unas ratios muy superiores a las de las promociones convencionales como demostramos más adelante. Las empresas tienen una expectativa alta de los canales de comunicación móviles por su gran alcance de población, su bajo coste y sus altas ratios de redención (Clickatell, 2003). Además, los canales móviles son vistos como inmediatos, automáticos, fidedignos, discretos y que permiten una llamada a la acción directa, imposible de encontrar en otros canales de comunicación (Clickatell, 2003).

Referente a su adopción por los consumidores, un artículo de MobileEntertainment. com de enero de 2012, basándose en un estudio de Juniper Research, augura que la cantidad de personas que utilizarán promociones móviles será de 600 millones a nivel mundial, mientras que las redenciones llegarán al $8 \%$ en 2016, 8 veces mayores a los 
ratios actuales (Green, 2012). Se espera que el mercado mundial de los cupones móviles en 2016 esté alrededor de los 43.000 millones de dólares, lo que significaría un incremento sustancial frente a los 5.400 de 2011 (Green, 2012).

Las promociones a través del móvil funcionan, tal y como se ha demostrado en varios estudios. Los patrones de respuesta de los consumidores que reciben cupones a través de sus teléfonos móviles aumentan sus visitas a un comercio (Kondo, Jian \& Shahriar, 2008). Un futuro del todo prometedor, según las conclusiones de los académicos en la materia y de los profesionales de empresas.

\subsection{Factores que ayudan a aumentar la efectividad en las promociones comerciales utilizando dispositivos móviles}

Sobre los factores principales que pueden ayudar a aumentar la efectividad en las promociones comerciales a través de dispositivos móviles, los resultados obtenidos en la literatura analizada y que consideramos que son relevantes y de suma importancia para el sector académico y para el empresarial, nos indican lo siguiente:

Momento en el que llega la promoción al usuario. Este factor es clave para el éxito, ya que impactar al cliente en el momento adecuado aumenta su interés y facilita la redención de la promoción comercial. Es mucho más sencillo poder utilizar un vale descuento para un café a las 10 de la mañana, cuando pasas delante de la cafetería, que si lo has visto en una revista la noche anterior, que obliga a actuar de una forma más previsora, recortando el cupón descuento y guardándolo para utilizarlo en otro momento. Una comunicación realizada en un momento equivocado puede provocar reacciones negativas en los consumidores e incluso rencor hacia la marca (Barnejee \& Dholakia, 2008).

Confianza en la marca/establecimiento. Si el consumidor se fía de la marca que envía la promoción y la valora, tiene más posibilidades de hacerla efectiva. No existe el freno que supone probar un nuevo producto o servicio y que obliga a realizar una oferta superior o dar otra ventaja adicional para vencerlo. Los consumidores que demuestran preferencias por una marca son menos sensibles a las promociones de otras marcas que aquellos que tienen preferencias moderadas por la misma marca (Ortmeyer, Lattin \& Montgomery, 1991) y las marcas más preferidas tienen redenciones superiores que aquellas que lo son menos (Bawa \& Shoemaker, 1987).

Creatividad de la oferta. La gran cantidad de promociones que llegan a un consumidor a través de todos los canales con los que interactúa y que le pueden impactar son tantas que si no se logra captar su atención con creatividad será imposible comenzar el proceso de compra. En este sentido, la variable funciona igual que para la comunicación publicitaria convencional. Tal y como afirman Kondo, Jian \& Shahriar (2008), la impresión de un correo y si será abierto o no por el destinatario dependerá principalmente de la línea del asunto; por este motivo, las palabras clave son el nombre de la tienda que favorece la identificación, los descuentos y los cupones como palabras a 
destacar en el asunto.

Facilidad de Redención. Cuanto más complicamos la vida a los consumidores para obtener la ventaja promocional, más difícil será obtener una respuesta positiva. Promociones en las que para acceder al premio deben seguirse varios pasos (recoger pruebas de compra, introducirlas en un sobre, enviarlas por correo...) complican las posibilidades de que un cliente cumpla todos los pasos y requisitos solicitados para obtener la oferta promocional (Banerjee \& Yancey, 2010), bajando el porcentaje de redención.

Bondad de la base de datos. En las promociones push, la fiabilidad y actualización de la base de datos son un elemento básico, ya que perder impactos por falta de depuración de la bases de datos o impactar a un cliente objetivo que pertenece a otro segmento del que se dirige la promoción, provoca, además de una bajada de la redención, malestar por parte de estos usuarios. A una mayor actualización e información disponible en la base de datos, más posibilidades de respetar a los consumidores, segmentar la oferta y enviar un mensaje más personalizado, lo que se traduce en un mayor éxito de la promoción. Algunos casos de estudio de consumidores japoneses son narrados por Ferris (2007), que anuncian el gran potencial de Internet móvil y las promociones basadas en una amplia base de datos de clientes potenciales vía data mining, segmentación y targetización, así como la medición de la efectividad de las campañas.

Valor de la oferta. Es obvio que cuanto más interesante sea la oferta para los consumidores, más posibilidades hay de seguir la promoción por parte de estos. En este punto los promotores han de tener en cuenta el balance entre una oferta motivante y los costes que supone, aconsejando controlar las promociones según lo que cuesten y que, preferiblemente, se autofinancien, no costando dinero a la organización (Kahneman \& Tversky, 1982) y ofreciendo un retorno de la inversión de la promoción positivo.

Simpleza de la promoción. Promociones complicadas provocan confusión en los consumidores y una bajada de su seguimiento. Si la promoción tiene mucho texto en sus bases seguramente pasarán dos cosas: que los consumidores no las sigan al no entenderlas o insatisfacción por alguna parte de ellos al tener unas expectativas que no coinciden con el resultado final obtenido con la promoción (Nunes \& Park, 2003).

Comunicación con target. La promoción es mucho más efectiva si está bien comunicada. Elementos de comunicación ayudarán a difundir más la promoción, consiguiendo una mayor redención al ser conocida por un número superior de consumidores (Banerjee \& Dholakia, 2008).

\section{Conclusión y recomendaciones futuras}

Como conclusión final, podemos señalar que la utilización de dispositivos móviles para efectuar promociones comerciales puede cambiar la forma en que las empresas se relacionan con sus clientes, abriéndoles nuevas posibilidades de interacción y sacando 
ventajas de éstas. Las reglas para el éxito en campañas de marketing móvil se fundamentan en el correcto desarrollo de la web móvil y en explotar sus posibilidades antes que los competidores, siendo la creatividad y el contenido claves, así como aprovechar las funcionalidades del dispositivo, buscar recomendaciones personales e incentivar los retos y premiarlos (The Chartered Institute of Marketing, 2011). Estas campañas se combinan con las de otros soportes y se lanzan simultáneamente en diferentes medios y plataformas a la vez, para conseguir una mayor notoriedad de la marca, producto o empresa anunciada (García, 2011).

Ahora el usuario tiene un rol activo, interactuando con las organizaciones a través de las funcionalidades de su smartphone o tableta, se relaciona con las marcas en movilidad y de manera inmediata, lo que invita a un replanteamiento de las herramientas promocionales tradicionales y a una mejor comprensión de las particularidades de este nuevo consumidor.

En este estudio se ha pretendido exponer los factores principales que pueden ayudar a aumentar la efectividad en las promociones comerciales a través de dispositivos móviles, según los resultados obtenidos de la literatura analizada y que consideramos relevantes e importantes para el sector académico y para el empresarial. Un posterior análisis más exhaustivo del tema se hace necesario a juzgar por los interesantes resultados obtenidos y será realizado de una manera empírica con análisis cuantitativos y cualitativos con el objetivo de comprobar de modo práctico los resultados obtenidos en el análisis teórico de la literatura sobre el tema.

\section{Referencias Bibliográficas}

BARNEJEE, S. Y DHOLAKIA, R.R. (2007): «Mobile advertising: does location based advertising work? » International Journal of Mobile Marketing, Vol. 3, December, pp. 68-74.

BARNEJEE, S. \& YANCEY, S. (2010): «Enhancing mobile coupon redemption in fast food campaigns». Journal of Research in Interactive Marketing, Vol. 4, No. 2, 97110.

BARWISE, P. Y STRONG C. (2002): «Permission-based mobile advertising». Journal of Interactive Marketing, Vol. 16 (1), 14-24.

BAWA, K. Y SHOEMAKER, R.W. (1987): «The effects of a direct mail coupon on brand choice behavior». Journal of Marketing Research, Vol. 24, 370-376.

CARMODY, B. (2001): Online Promotions: Winning Strategies and Tactics, New York, Wiley.

CLICKATELL. (2003): «SMS Marketing Guide». http://www.palowireless.com/sms/ docs/SMS market Guide mar03.pdf. Web visitada el 7/11/2011.

DAVID, F. (1989): «Perceived usefulness, perceived ease of use, and user acceptance of information technology». MIS Quarterly, Vol. 13, Num. 3, 319-340.

FERRIS, M. (2007): «Insights on mobile advertising, promotion and research». Journal of Advertising Research, Vol. 47, No. 1, March, 28-37. 
FORTÍN, D.R. (2000): «Clipping Coupons in Cyberspace: A Proposed Model of Behavior for Deal prone Consumers». Psychology \& Marketing, Vol. 17, June, 515-534.

GARCÍA MEDINA, I. (2011): «Marketing Digital Multimedia: Nuevos formatos y Tendencias». Revista Géminis, Ano. 2, No. 2, 37-45.

GODIN, S. (2001): El Marketing de Permiso, Barcelona, Editorial Granica.

GREEN, T. (2012): «600m regular mobile coupon users by 2016». http://www.mobileent.biz//news/read/600m-regular-mobile-coupon-users-by-2016. Web visitada el $5 / 1 / 2012$.

HEINONEN, K. Y STRANDVIK, T. (2011): «Consumer responsiveness to mobile marketing». International Journal of Mobile Communications, Vol. 5, Num. 6/2007. 603-617.

HOLMEN, E. «Got Recession? Mobile Marketing Goes Mainstream». Articles Mobile Marketing Association. 2008. http://mmaglobal.com/articles/got-recession-mobilemarketing-goes-mainstream. Web visitada el 12/11/2011.

JUNG, K. Y LEE, B.Y. (2010): «Online vs. offline Coupon redemption Behaviors». The International Business \& Economics Research Journal. December. 9-12.

Juniper Research. (2011): «Press Release: Mobile Coupon Redemption Rates to Reach 8\% Globally by 2016 as North American and Western European Users get Onboard». http://juniperresearch.com/viewpressrelease.php?pr=280. Web visitada el 24/3/2012.

KAHNEMAN, D. Y TVERSKY, A. (1982): The simulation heuristic Judgment under Uncertainty: Heuristics and Biases, New York, Cambridge University Press.

KAVASSALIS, P., SPYROPOULOU, N., DROSSOS, D., MITROKOSTAS, E. GIKAS, G., Y HATZISTAMATIOU, A. (2003): «Mobile permission marketing: Framing the market inquiry». International Journal of Electronic Commerce, Vol. 8 , No. 1, 55-79.

KONDO, F.N., JIAN, S.Z. Y SHAHRIAR, A. (2008): «Gradual changes in repeat customers' adoption behavior toward responses to mobile direct mail coupon promotions». Journal of Mobile Marketing, Volume: 3, Issue: 2, 15-24.

KONDO, F. Y NAKAHARA, M. (2007): «Differences in customers' responsiveness to mobile direct mail coupon promotions». International Journal of Mobile Marketing, Vol. 2, No. 2, 68-74.

KONDO, F.N., UWADAIRA, Y. Y NAKAHARA, M. (2007): «Simulating customer response to promotions: the case of mobile phone coupons». Journal of Targeting, Measurement and Analysis for Marketing, Vol. 17, No. 1, 57-67.

KOTLER, P. \& ARMSTRONG, G. (2001): Principles of Marketing, New Jersey, Pearson Prentice Hall, 13th. edition, 2010.

KTORIDOU, D., EPARNININDA, E. Y VRONTIS, D. (2007): «Technological and cultural aspects of the use of mobile marketing evidence from Cyprus». Ngmast 2007: International Conference on Next Generation Mobile Applications, Services and Technologies, 19-25.

LIVINGSTONE, A. (2004): «Smartphones and other mobile devices: The Swiss Army Knives of the 21st. century». Educause Quarterly, Núm 2.

NCH Marketing Services. (2010): 2010 Coupon Facts. https://www.nchmarketing. 
com/ResourceCenter/couponknowledgestream4a_ektid6611.aspx. Web visitada el $7 / 11 / 2011$.

LEPPÄNIEMI, M., Y KARJALUOTO, H. (2007): «Mobile Marketing: From Marketing Strategy to Mobile Marketing Campaign Implementation». International Journal of Mobile Marketing, Vol. 3, Issue 1, 50-62.

NESLIN, S.A. Y CLARKE, D.G. (1987): «Relating the brand use profile of coupon redeemers to brand and coupon characteristics». Journal of Advertising Research, Vol. 27, February/March, 23-32.

NOVIKOV, I. (2008): «Accuracy of Mobile Advertising Campaigns as Compared with Traditional Mass Media as an Advertising Medium». Articles Mobile Marketing Association. http://mmaglobal.com/articles/accuracy-mobile-advertising-campaignscompared-traditional-mass-media-advertising-medium. Web visitada el 27/12/2011.

NUNES J.C. Y PARK, W. (2003): «Incommensurate Resources: Not Just More of the Same». Journal of Marketing Research, Vol. 40, No. 1, 26-38

O'GUINN, T., ALLEN, C. Y SEMENIK, R.J. (1998): Advertising and Integrated Brand Promotion, Georgetown, Texas, USA, Ed. South-Western, College Publishing.

OORTMEYER, G., LATTIN, J.M. Y MONTGOMERY, D.B. (1991): «Individual Differences in Response to Consumer Promotions». International Journal of Research in Marketing, Vol. 8, 169-186.

RUIZ OLABUENAGA, J.I. (2007): Metodología de la investigación cualitativa, Bilbao, Publicaciones Universidad de Deusto.

SCHARL, A., DICKINGER, A. Y MURPHY, J. (2005): «Diffusion and success factors of mobile marketing». Electronic Commerce Research and Applications, Vol. 4, No. 2, 159-173.

SCHULTZ, D.E., ROBINSON, W.A. Y PETRISON, L.A. (1998): Sales promotion essentials: The 10 basic sales promotion technique and how to use them, Chicago, NTC Business Books.

The Chartered Institute of Marketing. (2011): It's not a phone: the future of Mobile Marketing, Maidenhead Berkshire, Ed. Cookham.

TSANG, M., HO, S.C., Y LIANG, T.P. (2004): «Consumer attitudes toward mobile advertising». International Journal of Electronic Commerce, Vol. 83, No. 3, 65-78.

WIEDMANN, K.P., WALSH, G. Y MITCHELL, V.W. (2001): «The Mannmaven: an agent for diffusing market information». Journal of Marketing Communications, Vol. 7, No. 4, 181-187.

YANG, H., LIU, H. Y ZHOU, L. (2012): «Predicting young Chinese consumers' mobile viral attitudes, intents and behavior». Asia Pacific Journal of Marketing and Logistics, Vol. 24, No. 1, 59-77. 